\title{
Successive structural conversion of lignin for chemical feedstock
}

\author{
Keigo Mikame and Masamitsu Funaoka \\ Graduate School of Bioresources, Mie University, SORST JST, 1577, Kurima-machiya, Tsu, Mie 514-8507, Japan \\ Fax: +81-59-231-9517, E-mail: mikame@bio.mie-u.ac.jp
}

\begin{abstract}
Although lignin is the most abundant natural phenolic polymer, its phenol activity is extremely low. Because most phenolic hydroxyl groups of lignin precursors were etherified in the biosynthetic process. In the present work, successive cleavage of ether linkages of lignin was carried out

The first step is the phase-separation process composed of phenols and concentrated acid. In the process, native lignin was modified by phenol derivatives to selectively grafted benzyl position, the most reactive sites, to give1,1-Bis(aryl) propane type lignin-based recyclable polymer (lignophenol) that has the original interunit linkage of lignin and have high phenolic content. The second step is cleavage of $C \beta$ aryl ether linkages by switching functions of lignophenol under the mild alkaline condition. The reaction mechanism was confirmed that with $\mathrm{C} \alpha$ phenolic nuclei (switching devise) having an $\mathrm{OH}$ ortho to the linking position with lignin, the phenoxy anions readily nucleophilically attack $C \beta$, so that $C \beta$ aryl ether linkages were cleaved. The third step is demethylation of the aromatic methoxyl groups. Demethylation of methoxyl group in lignophenol depolymerized products was carried out using boron tribromide. The methoxyl group of guaiacyl aryl coumaran was effectively demethylated to give catechol type aryl coumaran dimmer.
\end{abstract}

Key words: lignin, lignophenol, phase-separation system, methoxyl group, demethylation

\section{INTRODUCTION}

Recently plant biomaterials have attracted lots of attention as sustainable petroleum substitute again Especially polylactic acid as a biodegradable plastic from corn or sweet potato and biodiesel oil from soybean oil or palm oil have already been commercialized. But utilization of these plant biomaterials causes a price increase in food. Bio-ethanol from lignocellulosics which does not compete with food are proceeding. However these utilizations of plant biomaterials are conversion into materials for aliphatic compounds.

On the other hands, the aromatic compounds as petroleum substitute from plant biomaterials are hardly produced.

Lignin is the most abundant natural aromatic polymer and exists in plant cell walls as one of the major constituents. It serves as cement between wood fibers, a stiffening agent within fibers, and a barrier to the enzymatic degradation of the cell wall.

However, in contrast to the importance and potential of lignin in nature, lignin-based products have scarcely been in human life, because complicated structure and low reactivity of lignin. Although lignin is the most abundant natural phenolic polymer, its phenol activity is extremely low. Native lignin have only 0.1 to $0.2 \mathrm{~mol} / \mathrm{C}_{9}$ of phenolic hydroxyl groups. ${ }^{1,2}$ Because most phenolic hydroxyl groups of lignin precursors are etherified in the three steps biosynthetic process. $^{3,4}$

The first step is formation of methoxyl group. Lignin monomer is formed from phenylalanine or tyrosine via cinnamate pathway. In this pathway although $p$-coumaric acid is hydroxylated by $\mathrm{P}-450$ type hydroxylase, the hydroxylated compounds (caffeic acid and 5-hydroxyferulic acid) is immediately methylated by $O$-methyl taransferase. As a result one or two lignin-specific methoxyl groups are formed and phenolic hydroxyl groups are etherified.

The second step is polymerization of lignin precursors (coniferyl alcohol, sinapyl alcohol and $p$-coumaryl alcohol) via aryl ether linkage by radical coupling from enzymatic dehydrogenation.

The third step is formation benzyl aryl ether. Because a part of oligomeric products formed through radical coupling has quinonemethid structure, benzyl aryl ether linkage is formed by addition of adjacent phenolic hydroxyl groups.

Therefore many of phenolic hydroxyl groups are blocked by ether linkage. In other words, lignin has many latent phenolic hydroxyl groups.

In this study, we carried out successive cleavage of these ether linkages of lignin through the reverse pathway of lignin biosynthetic mechanism.

The first step is cleavage of benzyl aryl ether through the phase-separation system. In the 
process composed of phenols and concentrated acid, native lignin was modified by phenol derivatives to selectively grafted benzyl position, the most reactive sites, to give 1,1-Bis(aryl) propane type lignin-based recyclable polymer (lignophenol). ${ }^{5-8}$

The second step is cleavage of $C \beta$ aryl ether linkages by switching functions of lignophenol under the mild alkaline condition. ${ }^{8}$

The third step is demethylation of the aromatic methoxyl groups.

Trough these cleavage of ether linkages of lignin, we carried out activation and depolymerization of lignin for chemical feedstocks.

\section{EXPERIMENTAL}

2.1 Preparation of lignocresol through the phase-separation system

For solvation of lignin with phenol derivatives, $3 \mathrm{~mol} / \mathrm{C} 9$ (phenyl propane unit of lignin) of $p$-cresol dissolved in acetone was added to defatted wood meal (spruce and birch) and acetone was evaporated with stirring. Sulfuric acid $(72 \%, 10 \mathrm{ml} / \mathrm{g}$ wood) was added to the mixture and the vigorous stirring was continued at $30^{\circ} \mathrm{C}$ for $60 \mathrm{~min}$. The reaction mixture was rapidly poured to excess distilled water. The insoluble fraction was collected by centrifugation, washed with distilled water until neutral and lyophilized. The dried insoluble fraction was extracted with acetone. The acetone solution was then concentrated under reduced pressure and added dropwise to an excess amount of diethyl ether with stirring. The precipitated lignin derivative (lignocresol) was collected by centrifugation. Yields of lignocresol from spruce and birch were $27.5 \%$ of wood and $22.2 \%$ of wood respectively.

2.2 Depolymerization of lignocresol by switching functions

The dissolved lignocresol in $1.0 \mathrm{M} \mathrm{NaOH}$ solution was heating at $170^{\circ} \mathrm{C}$ for $1.0 \mathrm{hr}$. After cooling, reaction mixture was acidified by the $1.0 \mathrm{M} \mathrm{HCl}$. The precipitant was washed by de-ionized water and then dried. The molecular weight distribution of 2 nd derivatives from this lignocresol analyzed with size exclusion chromatography (SEC).

(SEC condition)

Column; Shodex GPC KF-801,802,803,804, Eluent; THF, Flow rate; $1.0 \mathrm{ml} / \mathrm{min}$, Temp.; $40^{\circ} \mathrm{C}$, Detect; $280 \mathrm{~nm}$, Standard: polystyrene standard

2.3 Isolation of main compound of 2 nd derivatives from lignocresol.

Second derivatives of lignocresol was fractionated by preparative SEC on Shimadzu LC-8A recycle preparative system equipped with Shimadzu SPD-10AUV/VIS detector. Shodex GPC KF-5002.5 (50 mm ID x $300 \mathrm{~mm}$ ) was connected and THF was used as an eluent (flow rate; $10 \mathrm{ml} / \mathrm{min}$ ). The low molecular weight fraction was purified by TLC after acetylation. The purified compound was identified by LC/MS,
${ }^{1} \mathrm{H}-\mathrm{NMR}$ and ${ }^{13} \mathrm{C}-\mathrm{NMR}$.

2.4 Demethylation of methoxyl group of $2 \mathrm{nd}$ derivatives from lignocresol.

Two $\mathrm{ml}$ of $1 \mathrm{M}$ boron tribromide dichloromethane solution was slowly added to a solution of $67 \mathrm{mg}$ of the low molecular weight fraction of 2 nd derivatives in dichloromethane at $-78^{\circ} \mathrm{C}$ for $1 \mathrm{hr}$ under nitrogen gas. ${ }^{9}$ The mixture was gradually warmed up to room temperature and stirred for $10 \mathrm{~min}$. Water was added to quench the reaction, and the mixture was extracted with ethyl acetate. The ethyl acetate extract was dried with $\mathrm{Na}_{2} \mathrm{SO}_{4}$, and the solvent was evaporated. The reaction mixture was purified by TLC. The purified compound was identified by LC/MS, ${ }^{1} \mathrm{H}-\mathrm{NMR}$ and ${ }^{13} \mathrm{C}-\mathrm{NMR}$.

\section{RESULTS AND DISCUSSION}

3.1 Cleavage of benzyl aryl ether through the phase-separation system

In the phase-separation system, lignin is greatly protected from the attack sulfuric acid through solvating with phenols. Lignin contacts with acid only in the interface between phenols and acid phase, and at that time, reactive benzyl alcohols and ethers mainly react with phenols. There is almost no any other secondary modification in

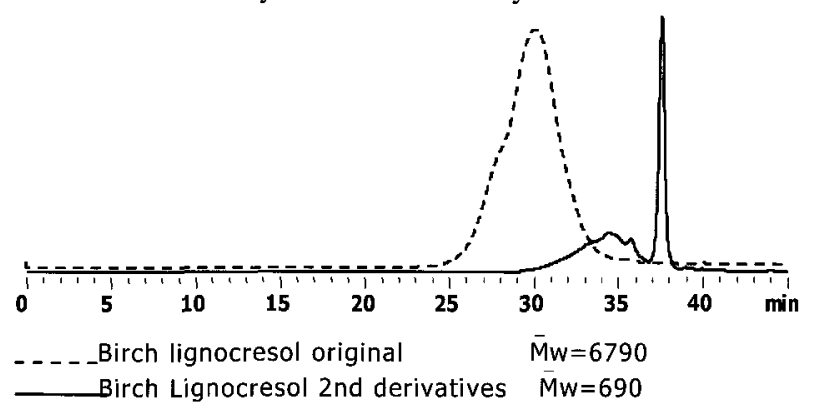

Fig. 1 SEC profiles of birch lignocresol and the 2nd derivatives

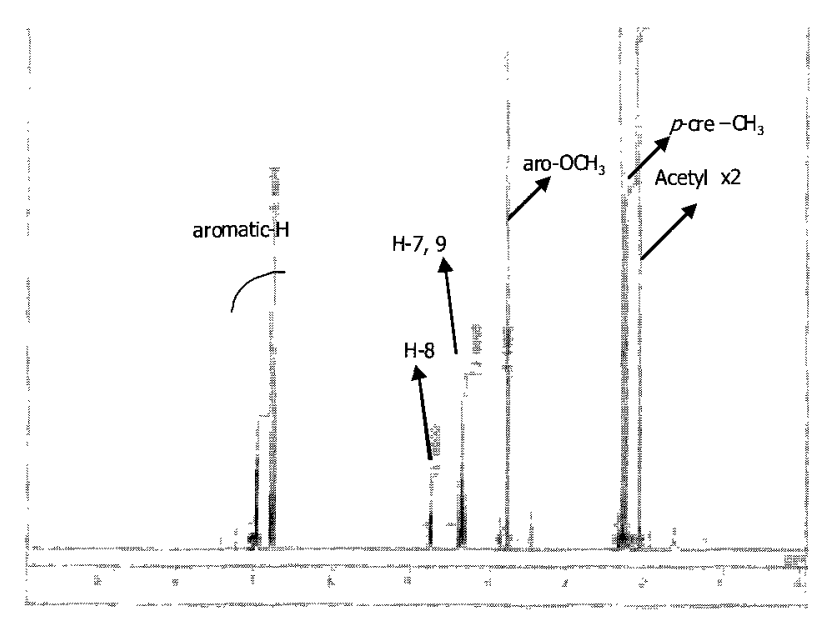

Fig. $2 \quad{ }^{1} \mathrm{H}-\mathrm{NMR}$ spectrum of acetylated guaiacyl aryl coumaran from birch lignocresol 2 nd derivatives. 
<smiles>COc1cc(C2c3cc(C)ccc3O[C@@H]2CO)cc(OC)c1O</smiles>

Fig. 3 The structures of acetylated guaiacyl and syringyl aryl coumarans from lignocresol 2nd derivatives.

this process. ${ }^{5-8}$ Therefore selective phenol grafting at Cl-positions gives 1,1-bis(aryl) propane type units in the lignin molecules, leading to both of improved phenolic functionality and decreased heterogeneity in structures reactivities. In this study, the amount of grafted cresol was $0.70 \mathrm{~mol} / \mathrm{C}_{9}$.

3.2 Cleavage of $C \beta$ ether by switching functions of lignophenol under the mild alkaline condition.

As shown Fig. 1, lignocresol from spruce and birch were significantly depolymerizated under alkaline treatment, indicated major peak at $37 \mathrm{~min}$ (about $\mathrm{Mw}=300$ ). This peak was fractionated by preparative SEC. The fractionated major peak compounds were purified by TLC after acetylation to prevent second modification. As a results of LC/MS analysis, one compound $(\mathrm{Mw}=370)$ was isolated from spruce lignocresol 2nd derivatives and two compounds $(\mathrm{Mw}=370$ and 400$)$ were isolated from birch lignocresol 2 nd derivatives. The structure of $\mathrm{Mw}=370$ compound was determined by ${ }^{1} \mathrm{H}$-NMR and ${ }^{13} \mathrm{C}$-NMR (Fig.2,3). This compound was guaiacyl aryl coumaran and the yield was $11.4 \%$ of spruce lignocresol. The structure of $\mathrm{Mw}=400$ compound was determined was syringyl aryl coumaran and the yield was $21.0 \%$ of birch lignocresol. The yield of guaiacyl aryl coumaran from birch lignocresol was $7.4 \%$. It was suggested that these aryl coumarans were formed by neighboring group participation reaction: the phenoxyide ion grafted phenols readily attacked the electron deficient $\beta$ position nucleophilically, resulting in the cleavage of $\beta$-aryl ether linkage. ${ }^{8}$ Therefore the higher yields of aryl coumaran from birch lignocresol indicated that birch lignocresol was considerably $\beta$-aryl ether linkage based liner type structure.

3.3 Demethylation of the aromatic methoxyl groups by lewis acid treatment.

Demethylation of methoxyl group in guaiacyl aryl coumaran was carried out using boron tribromide at $-78^{\circ} \mathrm{C}$ under nitrogen gas. In LC/MS analysis, ethyl acetate extractive of reaction mixture contained definitive peak having $\mathrm{m} / \mathrm{z}=270 \quad$ (Fig.4). This peak was presumed demethylated compound from guaiacyl aryl coumaran. Before structure confirmation, ethyl acetate extractive was acetylated, because catechol nucleus of putative compound was labile. In this LC/MS condition, the acetyl groups were eliminated under ionization. Thus it was confirmed that this compound contained three acetyl groups by both negative and positive mode of LC/MS. After TLC purification, acetylated compound was analyzed by ${ }^{1} \mathrm{H}-\mathrm{NMR}$ (Fig.5) and ${ }^{13} \mathrm{C}-\mathrm{NMR}$. As a results of these analysis, this compound was identified acetylated catechol aryl coumaran (Fig.6).

Therefore the methoxyl group of guaiacyl aryl coumaran was effectively demethylated to give catechol type aryl coumaran dimmer with boron tribromide treatment.

\section{CONCLUSION}

It is important to utilize lignin for oil substitution aromatic compounds because lignin is most abundant native aromatic compounds. However its phenol activity is extremely low. Because most phenolic hydroxyl groups of lignin precursors were etherified in the biosynthetic process. We carried out successive cleavage of these ether linkages of lignin and phenolic activation through the reverse pathway of lignin biosynthetic mechanism.

The native lignin was converted highly phenolic functional polymer (lignophenol) controlled molecular weight through the phase-separation treatment. The lignophenols was depolymerized to dimmer level with alkaline treatment. And the methoxyl group of the dimmer compound was demethylated with lewis acid treatment (Fig.7).

These successive cleavages of ether linkages of lignin is similar to decomposition mechanism of lignin in ecosystem. The decomposed lignin has several functions. Therefore the decomposed compounds from lignophenol also have high potentiality of aromatic compounds.

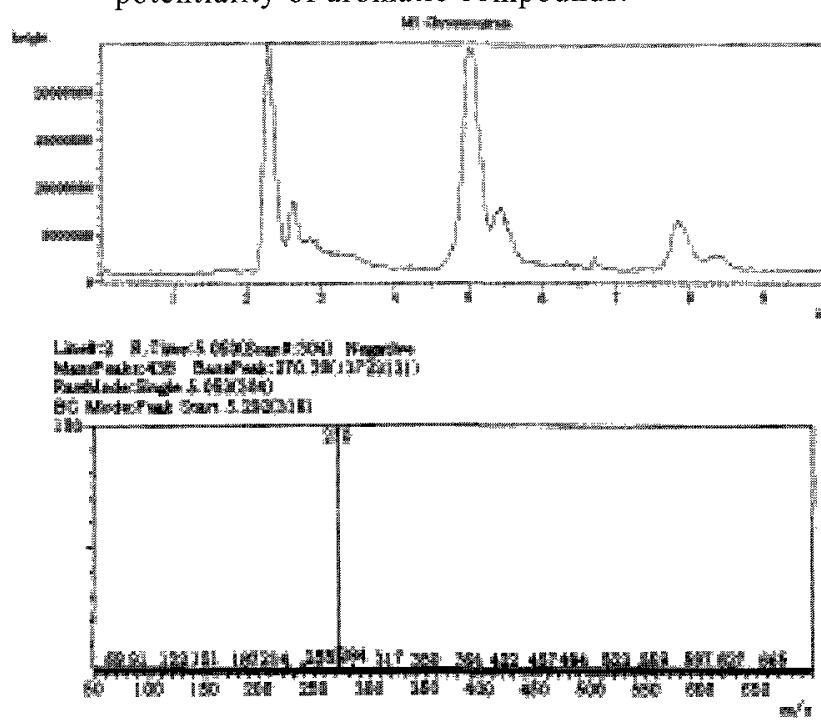

Fig. 4 Total ion chromatograph and mass spectrum of lignocresol 2nd derivatives low molecular weight fraction treated with $\mathrm{BBr}_{3}$. 


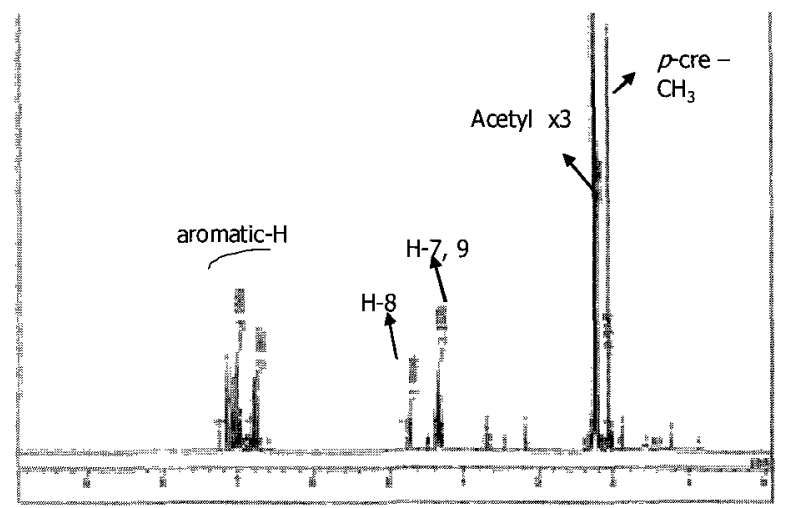

Fig. 5 'H-NMR spectrum of catechol aryl coumarans derived from guaiacyl aryl coumaran by lewis acid treatment.

\section{References}

[1] Y.-Z. Lai and M. Funaoka, J. Wood chemTechnol, 13, 43-54 (1993).

[2] Y.-Z. Lai and M. Funaoka, Holzforschung 47, 333-338 (1993).

[3] K. Freudenberg, Science, 148, 595-600 (1965).

[4] T. Higuchi, J. Biochem, 45, 515-528 (1958).

[5] M. Funaoka and I. Abe, Tappi Journal 72, 145-149(1989).

[6] M. Funaoka, M. Matsubara, N. Seki and S. Fukatsu, Biotechnol. Bioeng., 46, 545-552 (1995). [7] M. Funaoka and S. Fukatsu, Holzforschung 50, 245-252 (1996).

[8] M. Funaoka, Polymer International, 47, 277-290 (1998)

[9] Y. Hu, D. Kupfer, Drug Metabolism and Disposition, 30, 1035-1042 (2002)<smiles>Cc1ccc2c(c1)C(c1ccc(O)c(O)c1)C(CO)O2</smiles>

Fig. 6 The structures of catechol aryl coumarans derived from guaiacyl aryl coumaran by lewis acid treatment.

(Recieved June 6, 2008 ; Accepted September 5, 2008)

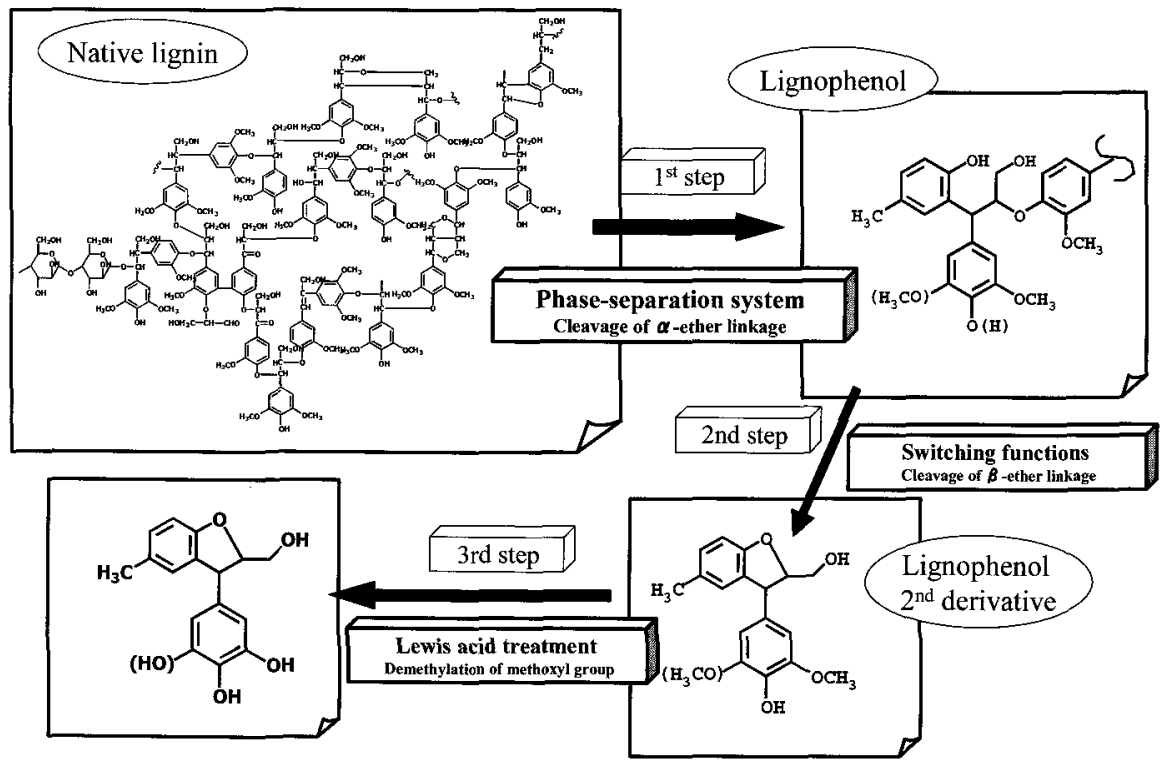

Fig. 7 Successive functionality control of lignin 